\title{
Tanshinone IIA alleviates hypoxia/reoxygenation induced cardiomyocytes injury via IncRNA AK003290/miR-124-5p signaling
}

\section{Liye Chen}

Peking University Health Science Centre

Lili Wei

Shihezi University School of Medicine

Qiongyang Yu

Peking University Health Science Centre

Haozhe Shi

Peking University Health Science Centre

George Liu ( $\square$ GeorgeLiu01@hotmail.com )

Peking University Health Science Centre

Research article

Keywords: Tanshinone IIA, hypoxia/reoxygenation, cardiomyocytes, long non-coding RNA, ceRNA

Posted Date: December 13th, 2019

DOI: https://doi.org/10.21203/rs.2.18747/v1

License: (c) (1) This work is licensed under a Creative Commons Attribution 4.0 International License. Read Full License 


\section{Abstract}

Objective

Acute myocardial infarction (AMI) is the leading cause of death and one of the heaviest healthy burden globally nowadays. Tanshinone IIA (TSA) is the major active compound extracted form Salvia miltiorrhiza Bunge possessing various of pharmacology activities. The present study aimed at investigating the role of TSA in AMI and underlying mechsniam.

Methods

Quantitative real-time PCR (qRT-PCR) and western blot assay were performed to detect the expression of genes and proteins. Cell apoptosis was detected by Annexin/V PI staining and flow cytometry. The amount of LDH, MDA and ROS were detected using commercial kits. FISH experiment was used to detect the expression of AK003290 in cardiomyocytes. Luciferace activity assay was performed to verify the interaction between AK003290 and miR-124-5p.

Results

We have shown that TSA decreased the apoptosis rate, the amount of LDH, MDA as well as ROS of cardiomyocytes. Meantime, it elevated mitochondrial membrane potential (MMP) which was decreased by $\mathrm{H} / \mathrm{R}$ treatment. It was also determined that miR-124-5p targets AK003290 directly. TSA up-regulated the expression of AK003290 and its function can be reversed by knock down of AK003290 as well as miR-124-5p overexpression.

Conclusion

TSA exerts the protective role against H/R induced apoptosis, oxidative and MMP loss of cardiomyocytes via regulating AK003290 and miR-124-5p signaling.

\section{Background}

Acute myocardial infarction (AMI) is characterized by the block of blood supply to the heart. It is the leading cause of death and one of the heaviest healthy burden globally nowadays [1,2]. Restoration of blood flow has been demonstrated as one of the most effective therapeutic methods to prevent the hearts from dysfunction or damage caused by imbalance of oxygen demand and supply. However, the recovery of blood supply termed as reperfusion together with reoxygenation will result in exacerbation of tissue injury as well as inflammatory responses which is called ischemia reperfusion (I/R) injury.

Tanshinone IIA (TSA) is the major active compound from the root extracts of Salvia miltiorrhiza Bunge, which is prescribed for treating cardiovascular disease in the pharmacopoeia of China as a traditional Chinese medicine (TCM) "Danshen". TSA has been shown to exert anti-angiogenic, antioxidant, antiinflammatory and antitumor activities. Increasing evidences have demonstrated the anti-tumor function 
of TSA in human cancers such as liver, cervical, gastric, colorectal, prostate, bladder, and breast cancers [3-10]. Moreover, due to its anti-oxidative and anti-inflammatory effects, TSA was indicated to possess cardio-protective effects. For instance, TSA has an anti-oxidative effect and prevents atherosclerosis by reducing vascular oxidative stress, inhibiting platelet aggregation, and protecting from endothelium damage [11-14]. Tanshinone IIA pretreatment protects myocardium against ischaemia/reperfusion injury through the phosphatidylinositol 3-kinase/Akt-dependent pathway in diabetic rats [15].

In the present study, we focused on the function of TSA on myocardial ischemic reperfusion injury. In vitro studies revealed that TSA alleviated the apoptosis, oxidative stress and MMP loss of cardiomyocytes subjected to hypoxia/reoxygenation (H/R) treatment. Mechanically, we found that TSA participates in the regulation of IncRNA AK003290/miR-124-5p signaling. These findings offer important insights into fundamental mechanisms underlying functions of TSA and IncRNAs, meantime, provided novel therapeutic targets for cardiac ischemic injury.

\section{Methods}

\section{Cell culture}

H9c2 cell were obtained from Cell bank of Chinese Academy of Sciences and cultured in DMEM (Gibco, CA, USA) supplemented with $10 \%$ fetal bovine serum (FBS, Gibco, CA, USA), $100 \mathrm{U} / \mathrm{mL}$ penicillin and $100 \mu \mathrm{g} / \mathrm{mL}$ streptomycin under $5 \% \mathrm{CO}_{2}$ at $37^{\circ} \mathrm{C}$ in a humidified atmosphere.

\section{Drug treatment}

TSA were purchased from National Institute for the Control of Pharmaceutical and Biological Products (> $99 \%$ purity, Beijing, China). Cells were treated with TSA $(0.5,1,5 \mu \mathrm{M})$ respectively 2 hours before inducing the hypoxia and during the hypoxia period.

\section{Plasmids construction}

MiR-124-5p mimic and mimic control as well as siRNA for AK003290 and the negative control are designed and synthesized by Genepharma (Shanghai, China). The wild and mutant region of AK003290 targeted by miR-124-5p were synthesized by Genepharma (Shanghai, China) and cloned into pGL3 luciferase reporter vectors (Promega, CA, USA).

\section{Flow cytometry}

The cultured $\mathrm{H} 9 \mathrm{c} 2$ cells were digested with trypsin, washed with cold PBS and dualstained with AnnexinV FITC/propidium iodide according to the manufacturer's instructions. Cell apoptosis was detected by flow cytometry on a BD FACSCalibur (Becton Dickinson, NJ, USA). 


\section{Reverse transcription and quantitative realtime PCR}

RNA extraction were performed with Trizol reagent (Invitrogen, CA, USA), precipitated with isopropanol, washed with $75 \%$ ethanol and dissolved in RNase free water. Reverse transcription were performed with $1 \mu \mathrm{g}$ RNA using the cDNA transcription kit (Transgen, USA). $20 \mathrm{ng}$ CDNA was used for qPCR to validate the expression of relative mRNA. It was determined by using SYBR green mix (Yisheng, Shanghai, China).

\section{Western blotting}

Cells were lysed on ice by lysis buffer (Beyotime, Shanghai, China), and the protein were extracted by centrifugation at $12000 \mathrm{~g}, 10 \mathrm{~min}, 4^{\circ} \mathrm{C}$. Protein lysates were loaded with $5 \times$ loading buffer on the SDSPAGE. After electrophresis, the gel was transferred to PVDF membrane. Followed with blocking with skim milk for 1 hour, protein bands on the PVDF membrane were incubated with relative primary antibodies at $4{ }^{\circ} \mathrm{C}$ overnight and the corresponding secondary HRP antibody at room temperature for 2 hour. The blots were visualized by ECL chemiluminiscence.

\section{RNA Pull-down assay}

Biotinylated miR-124-4p probe and the control probe were synthesized by Sangon Biotech (Shanghai, China). Probe-coated beads were generated by coincubating the probe with streptavidin-coated beads (Invitrogen, CA, USA) at $25^{\circ} \mathrm{C}$ for $2 \mathrm{~h}$. $\mathrm{H} 9 \mathrm{c} 2$ cells were collected, lysed and incubated with AK003290 or miR-124-5p probes overnight at $4^{\circ} \mathrm{C}$ respectively. Thereafter, the beads were eluted and the complex was purified with TRIzol (Takara, Dalian, China). Then the abundance of AK003290 and miR-124-5p was analyzed by qRT-PCR.

\section{LDH and MDA detection}

After reperfusion, the hearts were resected and homogenized in the cold phosphate buffer. After centrifuge at $3000 \mathrm{rpm}$ for $15 \mathrm{~min}$ the supernatant were collected for measurement at $-20^{\circ} \mathrm{C}$. The level of LDH as well as MDA were evaluated using commercial kits flowing their manufacturer's protocals (Jiancheng, Nanjing, China)

\section{ROS detection}

After I/R treatment, myocardial tissue or cardiomyocytes were collected and washed in PBS followed by co-culturing with $10 \mu \mathrm{M} \mathrm{DCFH-DA} \mathrm{(DCF-DA,} \mathrm{Shanghai,} \mathrm{Beyotime)} \mathrm{at} 37^{\circ} \mathrm{C}$ in an incubator for 20 min with gentle shaking in the dark. The mean fluorescence intensity (MFI) was evaluated using a flow cytometry (BD FACSCalibur, Becton Dickinson, NJ, USA). 


\section{Fluorescence in situ hybridization (FISH)}

Alexa Fluor 555-labeled AK003290 probes were designed and synthesized by RiboBio (Guangzhou, China). FISH experiment was carried out with a fluorescent in Situ Hybridization Kit (RiboBio, Guangzhou, China). $1 \times 10^{5}$ cells were was seed onto autoclaved glass slides and cultured for $24 \mathrm{~h}$. After fixing with $4 \%$ paraformaldehyde for 20 min followed by permeabilization with $0.5 \%$ Triton X-100 for $10 \mathrm{~min}$, the cells cultured at $37^{\circ} \mathrm{C}$ overnight. Finally, the slides were incubated with DAPI to stain cell nuclear and observed under a fluorescence microscope (Leica, Wetzlar, Germany).

\section{Luciferase activity assay}

The wild-type or mutant seed sequence at the predicted region of AK003290 and miR-124-5p were synthesized and cloned into the pGL3 Luciferase Reporter vectors (Promega, CA, USA) at the Kpnl and BamHI sites. $\mathrm{H} 9 \mathrm{c} 2$ cells were co-transfected with miR-124-5p mimics or mimic control, together with pGL3 vectors, which contained the WT or mut predicted binding region of AK003290. TRL-SV40 plasmid (Promega, CA, USA) was also transfected as a normalizing control. The cells were harvested for the detection of the activity of luciferase with the use of the Dual-Luciferase Assay (Promega, WI, USA) at $48 \mathrm{~h}$ following the transfection.

\section{Statistical analysis}

SPSS 20.0 software (SPSS Inc., Chicago, IL, USA) were used to analyze all data for statistical significance. All the data are presented as the means \pm SD. One-way ANOVA was used to assess the difference between multiple groups. Differences between two groups were analyzed by the Student's ttest. $\mathrm{P}<0.05$ was considered as statistical significance.

\section{Results}

\section{TSA alleviated $\mathrm{H} / \mathrm{R}$ induced apoptosis, oxidative stress and loss of mitochondrial membrane potential}

TSA has been reported to be capable of attenuating the I/R injury in cardiomyocytes. We first established $\mathrm{H} / \mathrm{R}$ model of cardiomyocyts to evaluated this function of TSA. As Fig. 1A showed, TSA of 30 and $60 \mu \mathrm{M}$ reduced the apoptosis of cardiomyocytes induced by H/R. In addition, MDA, LDH and ROS were detected to evaluate the oxidative stress of cardiomyocytes. We found that $\mathrm{H} / \mathrm{R}$ significantly elevated the level of MDA, LDH and ROS, meantime, TSA of 30 and $60 \mu \mathrm{M}$ notably reduced this elevation (Fig. 1B-D). Moreover, MMP was evaluated via JC-1 staining. As showed in Fig. 1E, TSA of 30 and $60 \mu \mathrm{M}$ elevated the MMP which was decreased by $\mathrm{H} / \mathrm{R}$ treatment. In order to further verify the effect of TSA on cell apoptosis, we detected the expression level of apoptotic proteins. It was indicated that $H / R$ promoted expression 
level of apoptotic protein such as bax, cytoplasma cyt-c, cleaved caspase3 while inhibited that of antiapoptotic protein bcl-2. 3 dose of TSA reversed these alteration induced by H/R (Fig. 1F).

\section{TSA promoted the expression of AK003290}

To explore novel mechanisms underlying the effect of TSA. We focused on the IncRNA which has attracted a lot of attention due to their various functions. We searched several IncRNAs that have been reported to play critical roles in the cardiovascular system diseases and evaluated their expression level after TSA treatment. The results indicated that TSA elevated the level of AK003290, MEG3, CASC7, HOTAIR while decreased that of ROR (Fig. 2A). Further, we selected and evaluated the expression of AK003290 after H/R and TSA treatment using qPCR and FISH assay. As Fig. 2B revealed, AK003290 was notably down-regulated by $H / R$ treatment. TSA significantly reversed the effect of $H / R$ (Fig. 2B, C).

\section{Knock down of AK003290 reversed the effect of TSA on H/R injury}

As AK003290 was down-regulated by $\mathrm{H} / \mathrm{R}$ and up-regulated by TSA treatment, we speculated that AK0032090 exerts the protective role against H/R injury in cardiomyocytes. We established the siRNA for AK003290. As expected, knock down of AK003290 reversed the effect of TSA (30 $\mu \mathrm{M})$ on apoptosis (Fig. 3A). Moreover, the same trend was found in the oxidative stress (Fig. 3B-D) and loss of MMP (Fig. 3E). Again, we detected the expression of apoptotic proteins. AK003290 knock down increased the expression level of bax, cytoplasma cyt-c, cleaved caspase 3 and reduced that of bcl- 2 in cardiomyocytes compare to TSA treatment group (Fig. 3F).

\section{AK003290 sponges miR-124-5p in cardiomyocytes}

As we know, IncRNAs are capable of sponging miRs to block their regulatory function on target genes. Here, we predicted the verified the miR targeting AK003290 to further elucidate the mechanism underlying the effect of AK003290. Figure 4A showed the targeting sequences between AK003290 and miR-124-5p. Luciferase activity assay indicated that miR-124-5p directly targets AK003290 in cardiomyocyte (Fig. 4B). Furthermore, the level of miR-124-5p was notably reduced in AK003290 knock down group (Fig. 4C). RNA pull down was carried out to detect whether AK003290 could directly bind to miR-124-5p endogenously. We found that biotin labeled miR-124-5p probe enriched the level of AK003290 which indicating the interaction between AK003290 and miR-124-5p (Fig. 4D). Moreover, the qPCR analyses further confirmed that miR-124-5p overexpression decreased the level of AK003290 while knock down of miR-124-5p increased that of AK003290 (Fig. 4D). Pearson analysis was finally carried out which indicated a negative correlation between miR-124-5p and AK003290 (Fig. 4E). 


\section{MiR-124-5p overexpression reversed the effect of TSA on $H / R$ injury}

To confirm whether miR-124-5p participates in the function of TSA on H/R injury. Rescue experiment was carried out. It was found that miR-124-5p overexpression reversed the effect of TSA on apoptosis (Fig. 5A). Moreover, the same trend was found in the oxidative stress (Fig. 5B-D) and loss of MMP (Fig. 5E). We further detected the expression of apoptotic proteins and found that miR-124-5p overexpression increased the expression level of bax, cytoplasma cyt-c and cleaved caspase 3 and reduced that of bcl-2 of cardiomyocytes in comparison to TSA treatment group (Fig. 5F).

\section{Discussion}

Apoptosis is well known to be the critical form of myocardial cells death induced by dysfunction of mitochondria and increase of lipid peroxides contributes mainly to $\mathrm{I} / \mathrm{R}$ injury. Deeper understanding of the mechanisms of cardiomyocyte apoptosis is critical to prevent heart injury and treat heart disease. In the present study, we detected apoptosis of cardiomyocytes using flow cytometry. To further evaluate the apoptosis condition of cardiomyocytes, we detected the MMP as well as apoptotic proteins including bax, cytoplasm cyt-c, bcl-2 and cleaved caspase3. It was found that TSA reduced the apoptosis of cardiomyocytes subjected to $\mathrm{H} / \mathrm{R}$ and also alleviated the oxidative stress and MMP loss induced by $\mathrm{H} / \mathrm{R}$ treatment.

TSA has been extensively studied in I/R injury not only in cardiovascular system but also in cerebral I/R injury. These findings proved the protective function of TSA on myocardial I/R injury to a great extent. However, the molecular mechanism underlying the effect of TSA is extremely complicated. It was reported that TSA participate in the regulation of classic signal pathways such as AMPKs/mTOR [16],

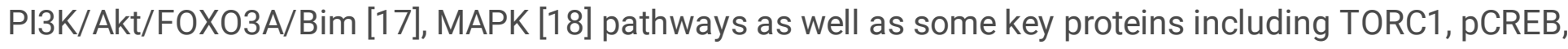
BDNF [19] and Na+/K+-ATPase[20]. However, the interaction between TSA and IncRNAs has been rarely studied.

Emerging evidences have shown that non-coding RNAs such as miRNAs and IncRNAs possess crucial roles in many biological processes. Previously, TSA was found to be capable of inhibiting miR-1 expression through p38 MAPK signal pathway in post-infarction rat cardiomyocytes [21]. In the present study, we identified that IncRNA AK003290 was down-regulated in cardiomyocyte subjected to H/R which indicated its protective role against H/R injury. Interestingly, TSA treatment elevated the level of AK003290. We speculated that AK003290 is involved in the effect of TSA on alleviating H/R injury. Further study revealed that knock down of AK020546 notably reversed the effect of TSA reducing cardiomyocyte apoptosis, oxidative stress and loss of MMP. These findings verified that TSA protected cardiomyocytes from H/R induced injury via up-regulating AK003290 expression.

It is well known that IncRNAs always act as sponges of miRNA thus participate in the regulation of their target genes that are involved in a common cellular function. Previous studies have reported that the APF, 
MALAT1, PFL mediates I/R injury through sponging miR-188-3p, miR-155/miR-203/miR-133 and let7d.respectively. Consequently, to further understand the critical role of AK003290 in cardiac I/R injury, we further explored the molecular mechanism by which AK003290 exerts its functions. Bioinformatic tools predicted that AK003290 is a potential target of miR-124-5p, luciferase activity assay verified our speculation indicating AK003290 as a direct target gene of miR-124-5p. Finally, rescue experiment confirmed our speculation indicating miR-124-5p as a downstream gene regulated by TSA. It is the first report demonstrating the function of TSA through IncRNA/miR signaling.

MiR-124-5p is a novel miRNA which has rarely been studied. We can known form the previous studies that miR-124-5p inhibits the growth of high-grade gliomas through posttranscriptional regulation of LAMB1 [22]. And low expression levels of microRNA-124-5p correlated with poor prognosis in colorectal cancer via targeting of SMC4. Here, we confirmed that miR-124-5p directly target AK003290. We performed rescue experiments and found both AK003290 knock down and miR-124-5p overexpression can reverse the effect of TSA. To further confirm the relation between AK003290 and miR-124-4p. Rescue experiment between AK003290 and miR-124-5p should be carried out.

\section{Conclusion}

In conclusion, we extended the understanding of the role of IncRNA in cardiac ischemia/reperfusion injury and provided a novel regulatory mechanism underlying the effect of TSA that is AK003290/miR-124-5p signaling. Our findings suggest novel biomarkers or potential therapeutic targets to treat ischemic heart diseases.

\section{Declarations}

\section{Ethics approval and consent to participate}

Not available.

\section{Consent to publish}

Not available.

\section{Availability of data and materials}

The datasets used and/or analysed during the current study are available from the corresponding author on reasonable request.

\section{Competing interests}

There is no competing interests in the present study.

\section{Funding}


This research was supported by National natural science foundation of China (30930037).

\section{Authors' Contributions}

study design: George Liu

experimental studies: Liye Chen, Lili Wei, Qiongyang Yu

Statistical analysis: Haozhe Shi,

Manuscript preparation: George Liu

\section{Acknowledgements}

Not available.

\section{Abbreviations}

LncRNA

Long non-coding RNA

AM

Acute myocardial infarction

TSA

Tanshinone IIA

$\mathrm{I} / \mathrm{R}$

ischemia reperfusion

$\mathrm{H} / \mathrm{R}$

hypoxia/reoxygenation

MMP

mitochondrial membrane potential

LDH

lactate dehydrogenase

MDA

Malondialdehyde

ROS

Reactive oxygen species

\section{References}

1. Pearcy RM, Mitchell SC, Smith RL: Beetroot and red urine. Biochem Soc Trans 1992, 20(1):22S.

2. Frank A, Bonney M, Bonney S, Weitzel L, Koeppen M, Eckle T: Myocardial ischemia reperfusion injury: from basic science to clinical bedside. Semin Cardiothorac Vasc Anesth 2012, 16(3):123-132. 
3. Lin CY, Chang TW, Hsieh WH, Hung MC, Lin IH, Lai SC, Tzeng YJ: Simultaneous induction of apoptosis and necroptosis by Tanshinone IIA in human hepatocellular carcinoma HepG2 cells. Cell Death Discov 2016, 2:16065.

4. Munagala R, Aqil F, Jeyabalan J, Gupta RC: Tanshinone IIA inhibits viral oncogene expression leading to apoptosis and inhibition of cervical cancer. CANCER LETT 2015, 356(2 Pt B):536-546.

5. Pan TL, Hung YC, Wang PW, Chen ST, Hsu TK, Sintupisut N, Cheng CS, Lyu PC: Functional proteomic and structural insights into molecular targets related to the growth inhibitory effect of tanshinone IIA on HeLa cells. PROTEOMICS 2010, 10(5):914-929.

6. Lin LL, Hsia CR, Hsu CL, Huang HC, Juan HF: Integrating transcriptomics and proteomics to show that tanshinone IIA suppresses cell growth by blocking glucose metabolism in gastric cancer cells. BMC GENOMICS 2015, 16:41.

7. Shan YF, Shen X, Xie YK, Chen JC, Shi HQ, Yu ZP, Song QT, Zhou MT, Zhang QY: Inhibitory effects of tanshinone II-A on invasion and metastasis of human colon carcinoma cells. ACTA PHARMACOL SIN 2009, 30(11):1537-1542.

8. Chiu SC, Huang SY, Chen SP, Su CC, Chiu TL, Pang CY: Tanshinone IIA inhibits human prostate cancer cells growth by induction of endoplasmic reticulum stress in vitro and in vivo. Prostate Cancer Prostatic Dis 2013, 16(4):315-322.

9. Chiu SC, Huang SY, Chang SF, Chen SP, Chen CC, Lin TH, Liu HH, Tsai TH, Lee SS, Pang CY et al: Potential therapeutic roles of tanshinone IIA in human bladder cancer cells. INT J MOL SCI 2014, 15(9):15622-15637.

10. Wang X, Wei Y, Yuan S, Liu G, Lu Y, Zhang J, Wang W: Potential anticancer activity of tanshinone IIA against human breast cancer. INT J CANCER 2005, 116(5):799-807.

11. Straka M, Straka-Trapezanlidis M, Deglovic J, Varga I: Periodontitis and osteoporosis. Neuro Endocrinol Lett 2015, 36(5):401-406.

12. Bahekar AA, Singh S, Saha S, Molnar J, Arora R: The prevalence and incidence of coronary heart disease is significantly increased in periodontitis: a meta-analysis. AM HEART J 2007, 154(5):830837.

13. Cai Y, Kurita-Ochiai T, Hashizume T, Yamamoto M: Green tea epigallocatechin-3-gallate attenuates Porphyromonas gingivalis-induced atherosclerosis. PATHOG DIS 2013, 67(1):76-83.

14. Chukkapalli SS, Velsko IM, Rivera-Kweh MF, Zheng D, Lucas AR, Kesavalu L: Polymicrobial Oral Infection with Four Periodontal Bacteria Orchestrates a Distinct Inflammatory Response and Atherosclerosis in ApoE null Mice. PLOS ONE 2015, 10(11):e143291.

15. Zhang Y, Wei L, Sun D, Cao F, Gao H, Zhao L, Du J, Li Y, Wang H: Tanshinone IIA pretreatment protects myocardium against ischaemia/reperfusion injury through the phosphatidylinositol 3-kinase/Aktdependent pathway in diabetic rats. DIABETES OBES METAB 2010, 12(4):316-322.

16. Zhang X, Wang Q, Wang X, Chen X, Shao M, Zhang Q, Guo D, Wu Y, Li C, Wang W et al: Tanshinone IIA protects against heart failure post-myocardial infarction via AMPKs/mTOR-dependent autophagy pathway. BIOMED PHARMACOTHER 2019, 112:108599. 
17. Zhang MQ, Zheng YL, Chen H, Tu JF, Shen Y, Guo JP, Yang XH, Yuan SR, Chen LZ, Chai JJ et al: Sodium tanshinone IIA sulfonate protects rat myocardium against ischemia-reperfusion injury via activation of PI3K/Akt/FOXO3A/Bim pathway. ACTA PHARMACOL SIN2013, 34(11):1386-1396.

18. Zhang Y, Zhang L, Chu W, Wang B, Zhang J, Zhao M, Li X, Li B, Lu Y, Yang B et al: Tanshinone IIA inhibits miR-1 expression through p38 MAPK signal pathway in post-infarction rat cardiomyocytes. CELL PHYSIOL BIOCHEM 2010, 26(6):991-998.

19. Liu L, Zhang X, Wang L, Yang R, Cui L, Li M, Du W, Wang S: The neuroprotective effects of Tanshinone IIA are associated with induced nuclear translocation of TORC1 and upregulated expression of TORC1, pCREB and BDNF in the acute stage of ischemic stroke. BRAIN RES BULL 2010, 82(3-4):228-233.

20. Wen PY, Li J, Lu BL, Liu J, Yang FZ, Zhou L, Luo H, Li WW, Zhou J: Tanshinone IIA increases levels of NeuN, protein disulfide isomerase, and $\mathrm{Na}+/ \mathrm{K}+$-ATPase and decreases evidence of microglial activation after cerebral ischemic injury. NEUROREPORT 2016, 27(6):435-444.

21. Zhang Y, Zhang L, Chu W, Wang B, Zhang J, Zhao M, Li X, Li B, Lu Y, Yang B et al: Tanshinone IIA inhibits miR-1 expression through p38 MAPK signal pathway in post-infarction rat cardiomyocytes. CELL PHYSIOL BIOCHEM 2010, 26(6):991-998.

22. Chen Q, Lu G, Cai Y, Li Y, Xu R, Ke Y, Zhang S: MiR-124-5p inhibits the growth of high-grade gliomas through posttranscriptional regulation of LAMB1. Neuro Oncol 2014, 16(5):637-651.

\section{Figures}

A

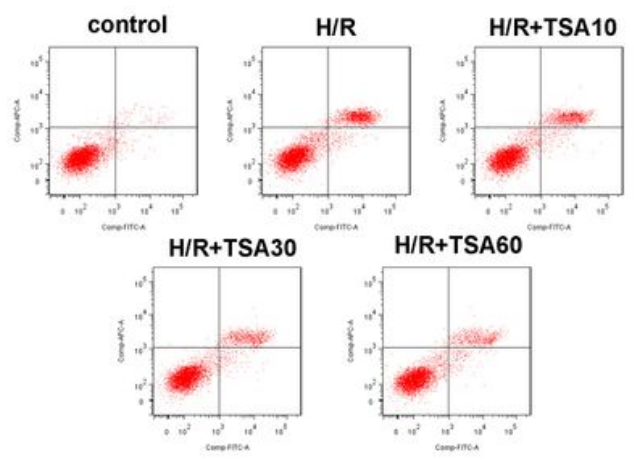

D

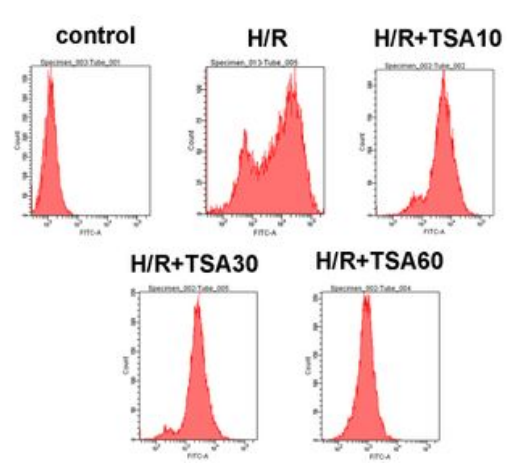

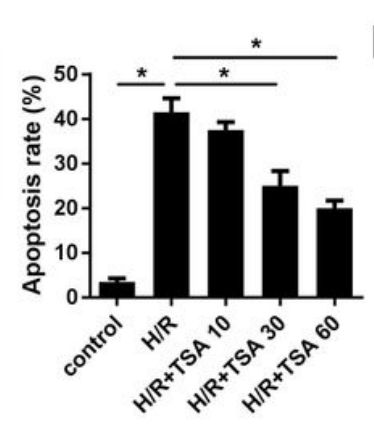

E

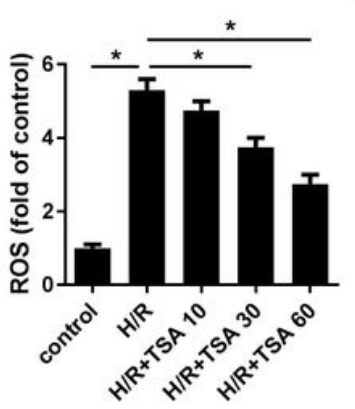

B
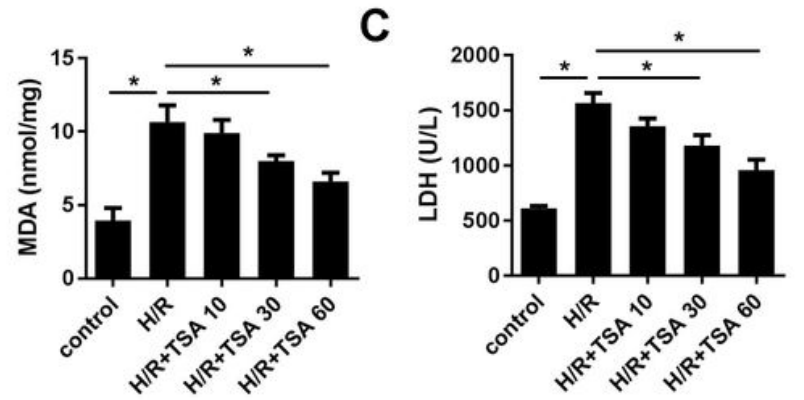

F

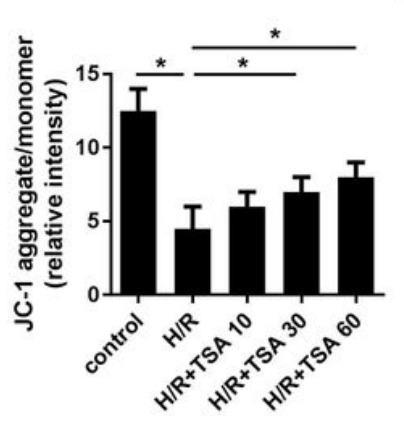

Figure 1 
TSA alleviated the apoptosis, oxidative stress and MMP loss induced by H/R. (A) Apoptosis of cardiomyocytes was evaluated using Annexin/VI-PI staining under different dose of TSA treatment. (B) MDA and LDH amount in cardiomyocytes were analyzed using commercial kit. (C) The ROS of cardiomyocytes was detected using commercial kit and flow cytometry. (D) Western blot was carried out to detect the apoptotic protein expression such as bax, cytoplasma cyt-c, cleaved caspase3 and bcl-2. ${ }^{*} \mathrm{p}$ \0.05.

A
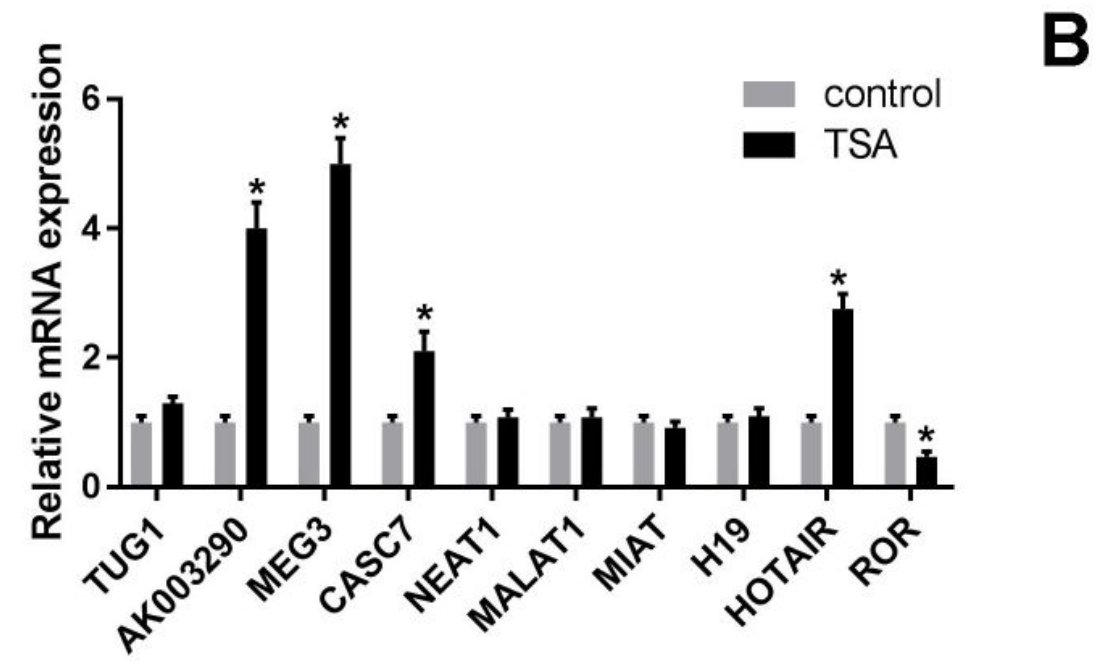

C

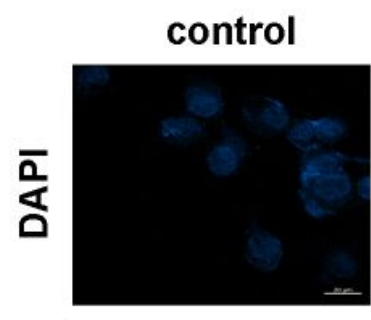

$H / R$

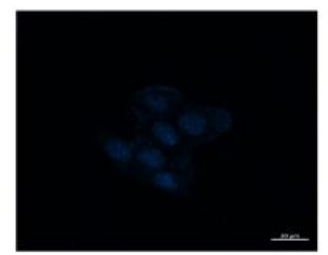

TSA10

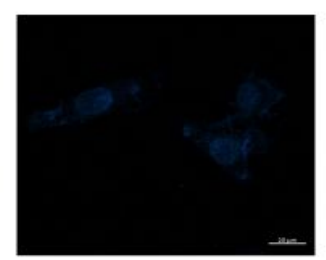

TSA30

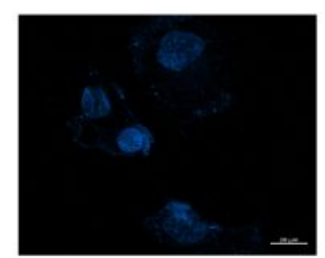

TSA60
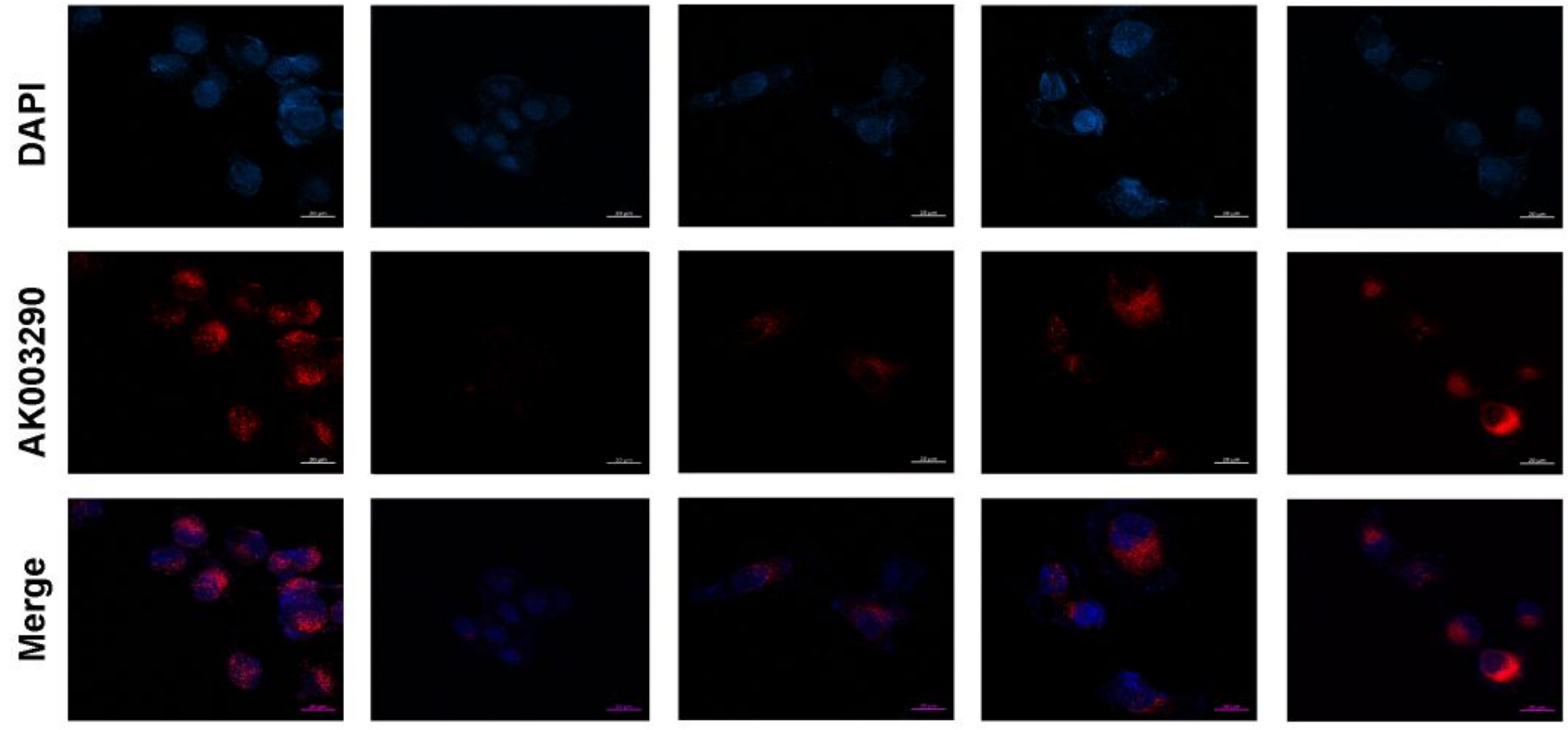

Figure 2

TSA promoted the expression level of AK003290. (A) qPCR was used to evaluate the expression of several IncRNAs correlated with heart disease. (B) qPCR was used to evaluate the expression of AK003290 in cardiomyocytes under different dose of TSA treatment. (C) FISH experiment was used to evaluate the expression of AK003290 under different dose of TSA treatment. *p®0.05. 
A
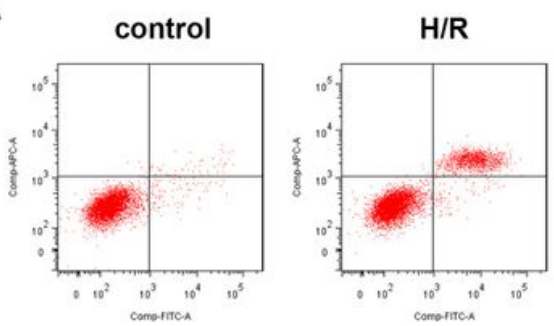

H/R+TSA+miR-nc

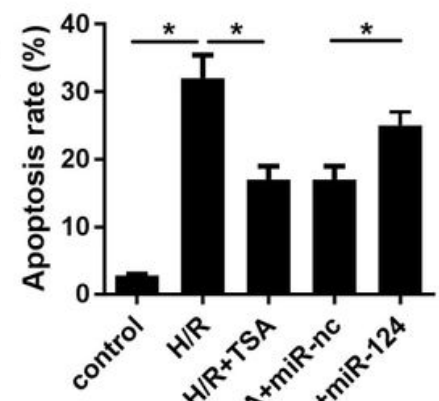

B

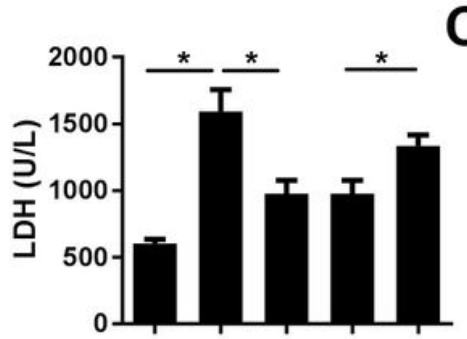

D

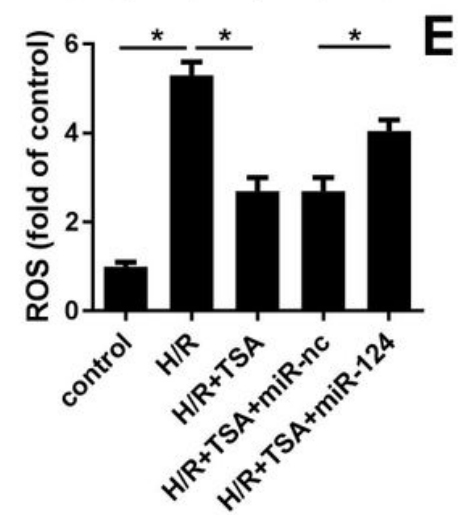

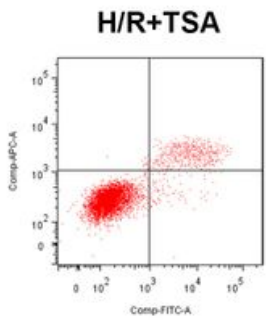
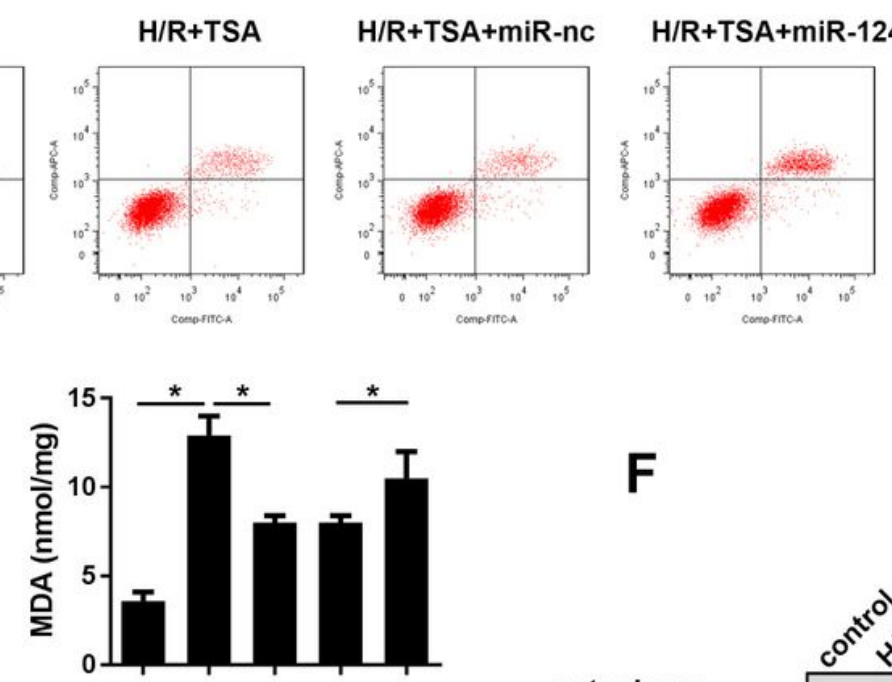

C
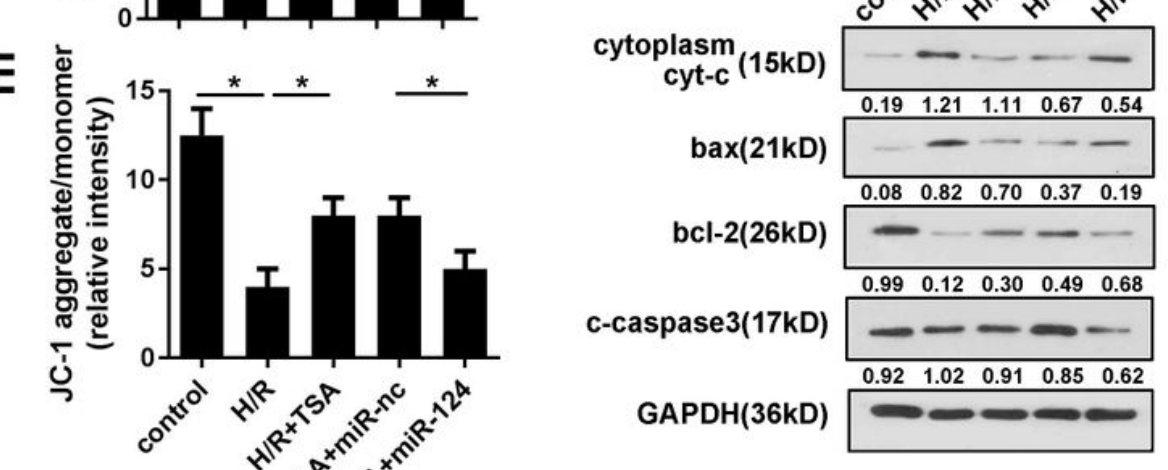

\section{Figure 3}

Knock down of AK003290 reversed the effect of TSA. (A) Apoptosis of cardiomyocytes was evaluated using Annexin/VI-PI staining under TSA treatment and si-AK003290 transfection. (B) MDA and LDH amount in cardiomyocytes were analyzed using commercial kit. (C) The ROS of cardiomyocytes was detected using commercial kit and flow cytometry. (D) Western blot was carried out to detect the apoptotic protein expression such as bax, cytoplasma cyt-c, cleaved caspase3 and bcl-2. *p $₫ 0.05$. 
A

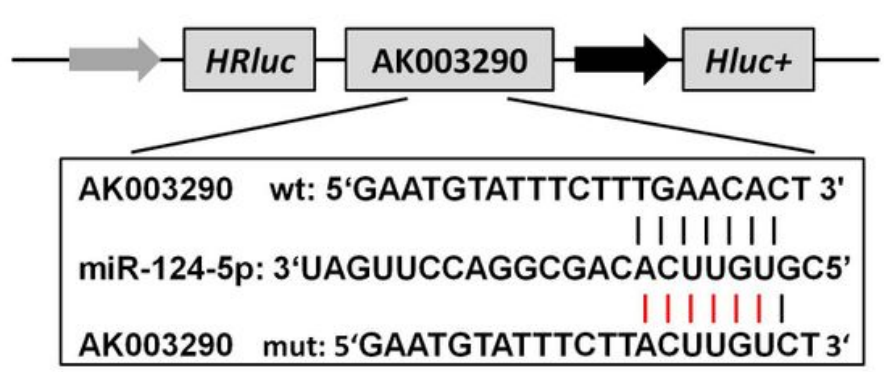

B

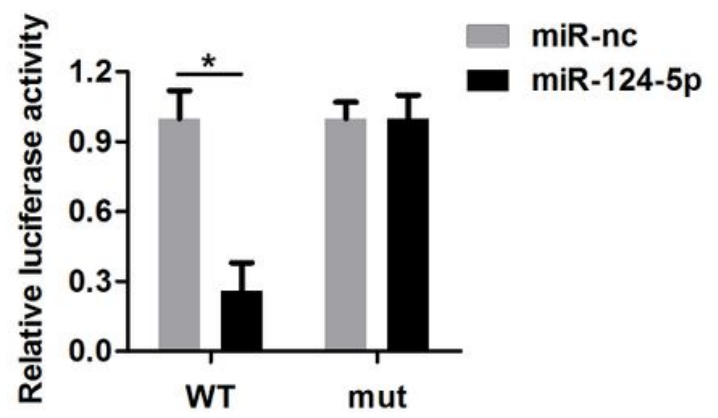

D

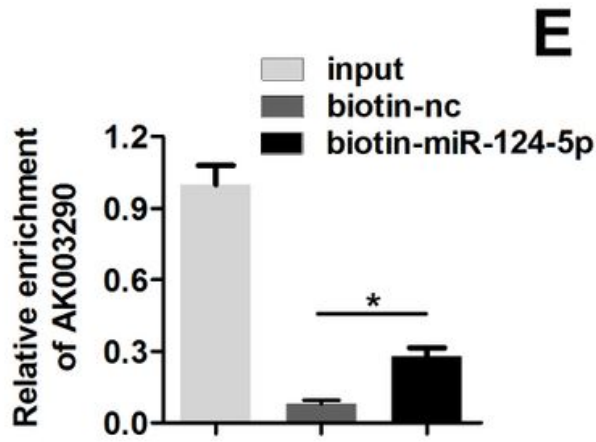

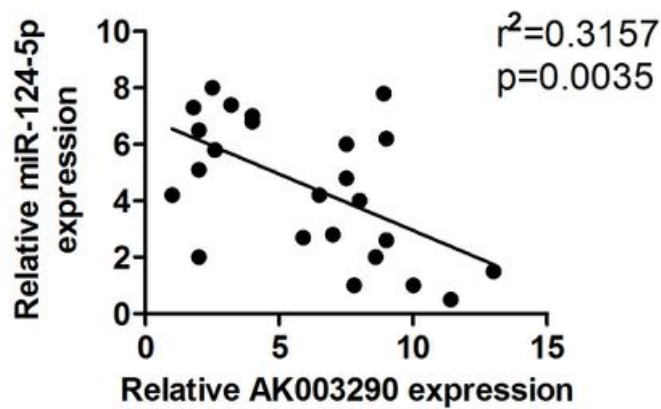

Figure 4

MiR-124-5p directly targetAK003290 in cardiomyocytes. (A) targeting region between miR-124-5p and AK003290. (B) Luciferase activity assay was carried out to verify whether miR-124-5p targets AK003290 in cardiomyocytes. (C) qPCR was performed to detect the expression of miR-124-5p. (C) RNA pull down using the probe of miR-124-5p was performed to detect the interaction between miR-124-5p and AK003290. (E) Pearson analysis was performed to investigate the correlation between circCadm1 and Pawr as well as miR-124-5p targets AK003290. *p®0.05. 
A
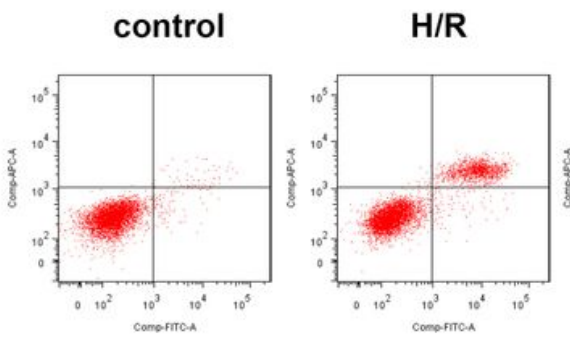

$H / R+T S A$

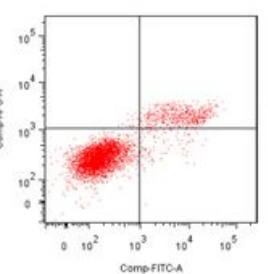

H/R+TSA+si-nc

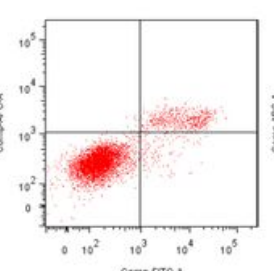

B

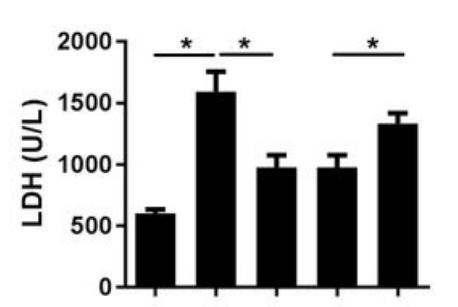

C

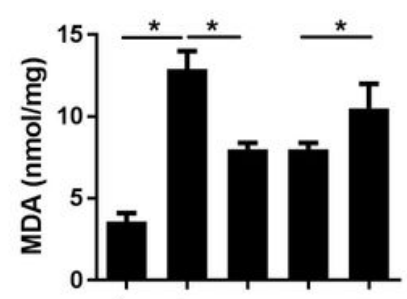

D

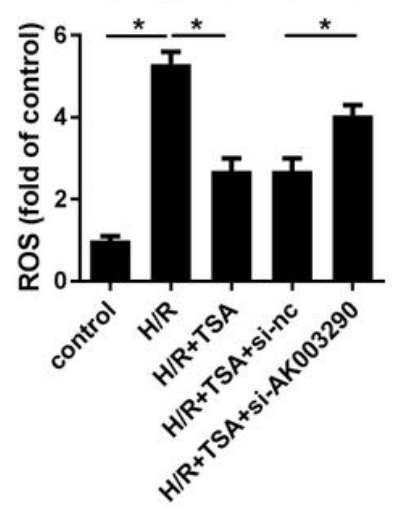

E

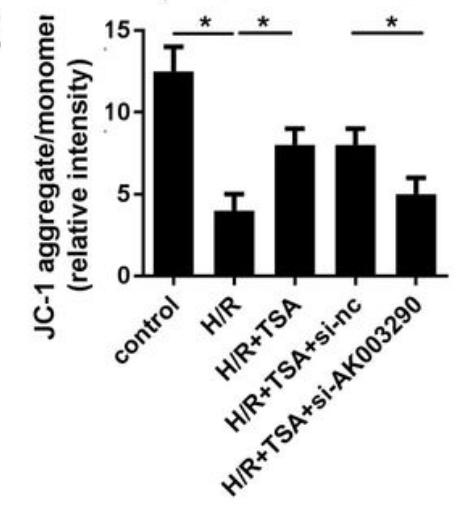

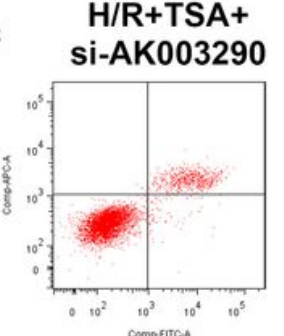

$\mathbf{F}$
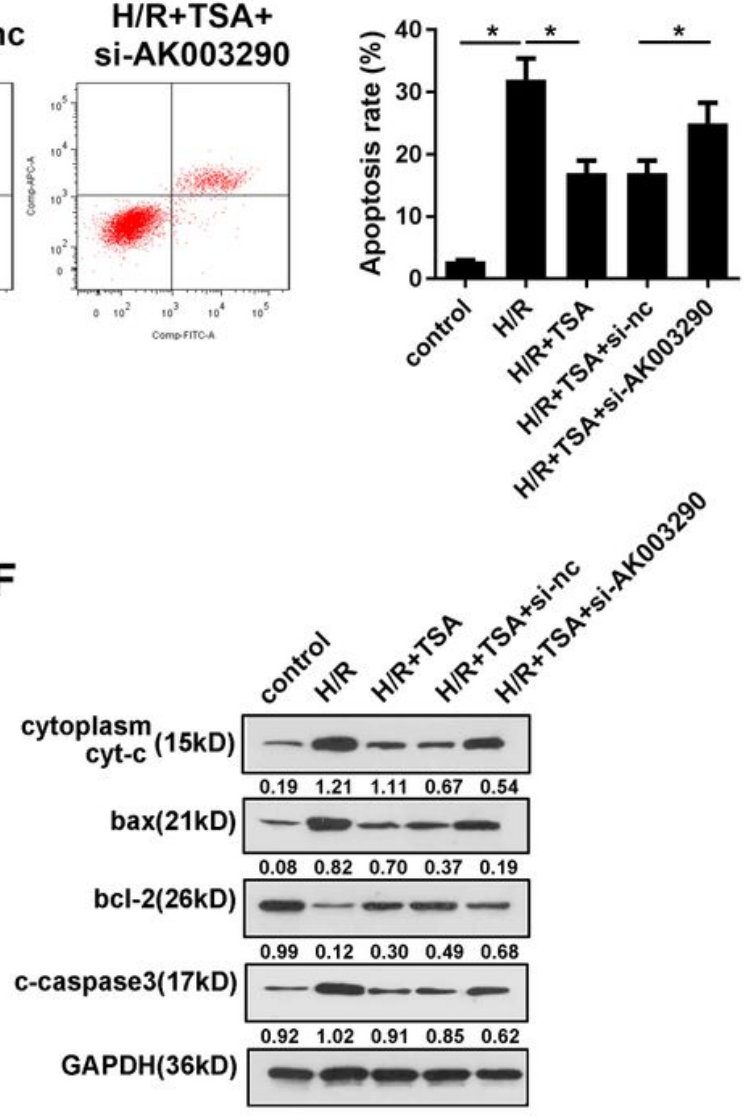

\section{Figure 5}

MiR-124-5p overexpression reversed the effect of TSA. (A) Apoptosis of cardiomyocytes was evaluated using Annexin/VI-PI staining under TSA treatment and miR-124-5p mimc transfection. (B) MDA and LDH amount in cardiomyocytes were analyzed using commercial kit. (C) The ROS of cardiomyocytes was detected using commercial kit and flow cytometry. (D) Western blot was carried out to detect the apoptotic protein expression such as bax, cytoplasma cyt-c, cleaved caspase3 and bcl-2. *pख0.05. 